\title{
MONOTONIC PARAREAL CONTROL FOR QUANTUM SYSTEMS
}

\author{
YVON MADAY*, JULIEN SALOMON ${ }^{\dagger}$, AND GABRIEL TURINICI $\ddagger$
}

\begin{abstract}
Following encouraging experimental results in quantum control, numerical simulations have known significant improvements through the introduction of efficient optimization algorithms. Yet, the computational cost still prevents using these procedures for high-dimensional systems often present in quantum chemistry. Using parareal framework, we present here a time parallelization of these schemes which allows to reduce significantly their computational cost while still finding convenient controls.
\end{abstract}

Key words. Quantum control, Monotonic schemes, Optimal control, Parareal scheme, time parallelization

AMS subject classifications. 49J20,68W10

1. Introduction. In the last decade, quantum control has witnessed significant developments including encouraging experimental results $([5,6,9,15,16,23,24,29$, $36])$. At the computational level $([7,25])$, the introduction of the monotonic algorithms of Krotov (by Tannor [31]), Zhu \& Rabitz ([37]) or the unified form of Maday \& Turinici ([21]) allows to design efficient methods to obtain laser fields controlling the molecular dynamics. On the other hand, parareal scheme (that stands for Parallelization in Real time) has shown a convenient efficiency in the case of the Schrödinger equation (see, e.g. $[2,33]$ ). In what follows, we combine these two approaches by using monotonic algorithms as inner loop of a time-parallelization procedure.

Let us first briefly present the model and the corresponding optimal control framework used in this paper. Consider a quantum system described by its wavefunction $\psi(x, t)$, also called state in what follows. Here " $x^{\prime \prime} \in \Omega$ denotes the relevant spatial coordinates (the symbol $x$ will often be omitted in what follows for reason of simplicity). The operator $V(x)$ is the potential part. The dynamics of this system is characterized by its internal Hamiltonian:

$$
H(x)=H_{0}+V(x) .
$$

In this equation $H_{0}$, the kinetic part, could be

$$
H_{0}=-\frac{1}{2} \sum_{n=1}^{p} \frac{1}{m_{n}} \Delta_{r_{n}}
$$

where $p$ is the number of particles considered, $m_{n}$ their masses and $\Delta_{r_{n}}$ the Laplace operator with respect to their coordinates.

A way to control such a system is to light it with a laser pulse. We denote by $\varepsilon(t)$ the intensity of this control field. The contribution of this parameter is taken into account by introducing a perturbative term in the Hamiltonian which then reads

\footnotetext{
*Laboratoire J-L. Lions, Université P. \& M. Curie, Paris, France (maday@ann.jussieu.fr) \& Division of Applied Maths, Brown University, Providence RI.

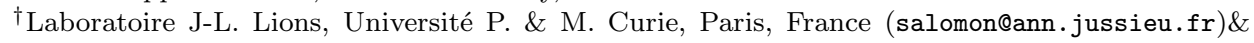
CEREMADE, Université Paris Dauphine, Pl. du Maréchal Lattre de Tassigny, 75775 Paris Cedex 16, France (salomon@ceremade.dauphine.fr).

$\ddagger$ CEREMADE, Université Paris Dauphine, Pl. du Maréchal Lattre de Tassigny, 75775 Paris Cedex 16, France (gabriel.turinici@dauphine.fr).
} 
$H(x)-\mu(x) \varepsilon(t)$. The evolution of $\psi^{\varepsilon}(x, t)$ is governed by the Schrödinger equation (we work in atomic units, i.e. $\hbar=1$ ):

$$
\left\{\begin{aligned}
i \frac{\partial}{\partial t} \psi^{\varepsilon}(x, t) & =(H(x)-\mu(x) \varepsilon(t)) \psi^{\varepsilon}(x, t) \\
\psi^{\varepsilon}(x, 0) & =\psi_{\text {init }}(x),
\end{aligned}\right.
$$

where $\psi_{\text {init }}$ is the initial condition for $\psi^{\varepsilon}$ subject to the constraint:

$$
\left\|\psi_{\text {init }}\right\|_{L^{2}(\Omega)}=1 \text {. }
$$

Since $H$ is self-adjoint, from (1.3), the norm of the state is constant with respect to the time. In the numerical simulations, the ground state, i.e. a unitary eigenvector of $H$ associated with the lowest eigenvalue, is generally taken as initial condition.

The optimal control framework can then be applied to this bilinear control system to design relevant control fields. The quality of the pulse is evaluated via a cost functional. A general example of such a function is:

$$
J(\varepsilon)=\left\|\psi^{\varepsilon}(T)-\psi_{\text {target }}\right\|_{L^{2}(\Omega)}^{2}+\int_{0}^{T} \alpha(t) \varepsilon^{2}(t) d t,
$$

where $T$ is the total time of control, $\alpha$ a positive function and $\psi_{\text {target }}$ a target state which has to be reached.

At the minimum of the cost functional $J$, the Euler-Lagrange critical point equations are satisfied; a standard way to write these equations is to use a Lagrange multiplier $\chi^{\varepsilon}(x, t)$ called adjoint state. The following critical point equations are thus obtained [37]:

$$
\begin{aligned}
& \left\{\begin{array}{l}
i \frac{\partial}{\partial t} \psi^{\varepsilon}(x, t)=(H(x)-\varepsilon(t) \mu(x)) \psi^{\varepsilon}(x, t) \\
\psi^{\varepsilon}(x, 0)=\psi_{\text {init }}(x)
\end{array}\right. \\
& \left\{\begin{array}{l}
i \frac{\partial}{\partial t} \chi^{\varepsilon}(x, t)=(H(x)-\varepsilon(t) \mu(x)) \chi^{\varepsilon}(x, t) \\
\chi^{\varepsilon}(x, T)=\psi_{\text {target }}(x)
\end{array}\right. \\
& \alpha(t) \varepsilon(t)=-\operatorname{Im}\left\langle\chi^{\varepsilon}(t)|\mu| \psi^{\varepsilon}(t)\right\rangle,
\end{aligned}
$$

where $A$ is an operator on $L^{2}(\Omega)$ and $\langle f|A| g\rangle=\int_{\Omega} \overline{f(x)} A(g)(x) d x$.

Numerous optimization procedures exist to compute iteratively sequences $\left(\varepsilon^{k}\right)_{k \in \mathbf{N}}$ that approximate the solution of (1.6)-(1.8). The common feature of these algorithms is that they involve repeated resolutions of Schrödinger equations (1.6) and (1.7), which induce a heavy computational time cost. Depending on their order, the mere computation of $\varepsilon^{k}$ can also be time consuming due to the high nonlinearity of the cost functional. In order to reduce the computation time, some time parallelization of the resolution of (1.6)-(1.8) can be done. A standard method consists in subdividing the interval $[0, T]$ into subintervals and to compute iteratively the corresponding adequate initial conditions for parallel resolution on each subinterval. This approach is also the base of the multiple shooting methods (see [22] Sec. 17.1, and, e.g. [8]). In [10] a comparison between these method shows that the parareal algorithms can be recast as a multiple shooting algorithm where the Jacobian matrix is approached by a finite difference method on a coarse grid. 
Another body of related literature was introduced in the pioneering works of Hackbush (see $[11,12]$ and also $[14,19,35])$. The parareal method corresponds to a two-level multigrid with a larger coarsening rate and an unusual smoother which corresponds to a single phase of a bicolor relaxation scheme.

Such time parallelization procedures have already been associated to optimization procedures to tackle control problems, e.g. in the case of ordinary differential equations [13] or linear control of hyperbolic [17] or parabolic evolution equations [4, 34]. On the contrary, we present here a new method to treat the bilinear optimal control of the Schrödinger equation (1.3), and consider a particular decomposition of $J$ in sub-cost functionals corresponding to the time subdivision. In this framework, an iterative optimization procedure is designed that converges to a critical point of $J$.

The paper is organized as follows: parareal optimal control settings corresponding to our quantum control problem is presented in section 2. In section 3 , we give an algorithm achieving a parallel in time optimization. We prove the convergence of this procedure towards a critical point of a discrete version of the cost functional $J$ in section 4 and we finally give some numerical results in section 5 .

2. Parareal control setting. Throughout this section the control field $\varepsilon$ is either a function of $L^{2}([0, T])$ or its corresponding time discretization.

2.1. Main features of the parallelization. Following Lions et al. ([18]), we now introduce the necessary concepts and tools involved in the time parallelization proposed by the parareal approach.

2.1.1. Subdivision of $[0, T]$ and virtual controls. Let $N \geq 1$ be an integer. In order to design time parallelized optimization procedure, we introduce a subdivision of $[0, T]$ :

$$
[0, T]=\cup_{\ell=0}^{N-1}\left[T_{\ell}, T_{\ell+1}\right]
$$

with $T_{0}=0$ and $T_{N}=T$. Consider also a set $\Lambda=\left(\lambda_{\ell}\right)_{\ell=1, \ldots, N-1} \in\left(L^{2}(\Omega)\right)^{N-1}$. In what follows, $\Lambda$ will be called the set of virtual controls. For notational simplicity, we will also denote by $\lambda_{0}$ the initial state $\psi_{\text {init }}$ and by $\lambda_{N}$ the target state $\psi_{\text {target }}$. The resolution of (1.3) is now substituted by the resolution of the $N$ problems:

$$
\left\{\begin{aligned}
i \frac{\partial}{\partial t} \psi_{\ell}^{\varepsilon_{\ell}}(x, t) & =\left(H(x)-\varepsilon_{\ell}(t) \mu(x)\right) \psi_{\ell}^{\varepsilon_{\ell}}(x, t) \\
\psi_{\ell}^{\varepsilon_{\ell}}\left(x, T_{\ell}\right) & =\lambda_{\ell}(x), \quad \ell=0, \ldots, N-1,
\end{aligned}\right.
$$

where $\varepsilon_{\ell}$ is the restriction of $\varepsilon$ to $\left[T_{\ell}, T_{\ell+1}\right]$ (with $\ell=0, \ldots, N-1$ ). By $(2.2), \psi_{\ell}^{\varepsilon_{\ell}}$ also depends on $\lambda_{\ell}$. In order to simplify the notations, we omit this dependence. The solution of (2.2) coincides to that of (1.3) if and only if :

$$
\forall \ell=0, \ldots, N-1, \quad \psi_{\ell}^{\varepsilon_{\ell}}\left(x, T_{\ell+1}\right)=\lambda_{\ell+1}(x) .
$$

The parareal framework provides different methods to iteratively compute a solution $\Lambda^{*}$ of $(2.2)-(2.3)$.

2.1.2. Coarse and fine propagators. Suppose that the numerical simulation of (1.3) can be realized both by a coarse propagator and a fine propagator. Because the use of the coarse propagator is considered to be cheap, it can be used for the resolution of (1.3) over the whole interval of control $[0, T]$. On the contrary, the fine propagator will only be used for parallel resolutions on $\left[T_{\ell}, T_{\ell+1}\right]$. 
The analysis of the algorithm presented below requires that the $L^{2}$-norm of the finely approximated solution be constant with respect to the time (as it is the case for the exact solution of (1.3)). Because of its numerical accuracy, we choose to consider the Strang-second-order split-operator solver $([3,30])$, that fulfills this property. Let us present it in some detail.

Consider two parameters of the time discetization $n$ and $\delta t=\frac{T}{n}$, and define $n_{0}=0<$ $n_{1}<\ldots<n_{\ell}<\ldots<n_{N}=n$ the time indexes associated with $\left(T_{\ell}\right)_{\ell=0, \ldots, N}$. Let us also introduce, for $\ell=0, \ldots, N-1, j=n_{\ell}, \ldots, n_{\ell+1}-1$, the discretized control field $\varepsilon_{\ell, j}$ and for $\ell=0, \ldots, N-1, j=n_{\ell}, \ldots, n_{\ell+1}$, the discretized state $\psi_{\ell, j}^{\varepsilon_{\ell}}$ that stand respectively for approximations of $\varepsilon_{\ell}(j \delta t)$ and $\psi_{\ell}^{\varepsilon_{\ell}}(j \delta t)$. The time-discretization of (2.2) is given by:

$$
\left\{\begin{aligned}
\psi_{\ell, j+1}^{\varepsilon_{\ell}} & =e^{\frac{H_{0} \delta t}{2 i}} e^{\frac{V-\mu \varepsilon_{\ell, j}}{i} \delta t} e^{\frac{H_{0} \delta t}{2 i}} \psi_{\ell, j}^{\varepsilon_{\ell}}, \quad j=n_{\ell}, \ldots, n_{\ell+1}-1 \\
\psi_{\ell, n_{\ell}}^{\varepsilon_{\ell}} & =\lambda_{\ell}, \quad \ell=0, \ldots, N-1 .
\end{aligned}\right.
$$

We refer the reader to [20] for additional details about the corresponding full discretization.

REMARK 1. Though we focus on the Strang-second-order split-operator scheme, the methodology presented in this paper can be adapted to other time discretizations. Indeed, the analysis done below only requires that the norm of the wavefunction is preserved during the propagation.

2.1.3. Parareal strategy. Parareal algorithms aim at computing in parallel on each subinterval the solution of evolution equations such as (1.3). To do this, they propose various iterative methods to update the virtual controls after each parallel propagations. The purpose of what follows is to define an update algorithm that allows to couple parareal approach with an optimization procedure of quantum control.

2.2. Parareal cost functionals. Let $\Lambda=\left(\lambda_{\ell}\right)_{\ell=1, \ldots, N-1}$ be a set of virtual controls and $\left(\psi_{\ell}^{\varepsilon_{\ell}}\right)_{\ell=0, \ldots, N-1}$ the corresponding time discretized solutions of the parallel propagations (2.4), with $\psi_{\ell}^{\varepsilon_{\ell}}=\left(\psi_{\ell, j}^{\varepsilon_{\ell}}\right)_{j=n_{\ell}, \ldots, n_{\ell+1}}$. In order to design a formulation combining optimal control and parareal framework, let us also consider the following cost functional:

$$
J_{\|}(\varepsilon, \Lambda)=\sum_{\ell=0}^{N-1} \beta_{\ell}\left\|\psi_{\ell, n_{\ell+1}}^{\varepsilon_{\ell}}-\lambda_{\ell+1}\right\|_{L^{2}(\Omega)}^{2}+\delta t \sum_{\ell=0}^{N-1} \sum_{j=n_{\ell}}^{n_{\ell+1}-1} \alpha_{j} \varepsilon_{\ell, j}^{2},
$$

where $\beta_{\ell}=\frac{n}{n_{\ell+1}-n_{\ell}}$ and $\alpha_{j}$ is an approximation $\alpha(j \delta t)$. This cost functional can be decomposed as follows:

$$
J_{\|}(\varepsilon, \Lambda)=\sum_{\ell=0}^{N-1} \beta_{\ell} J_{\ell}\left(\varepsilon_{\ell}, \lambda_{\ell}, \lambda_{\ell+1}\right),
$$

where $J_{\ell}$ are the parareal cost functionals:

$$
J_{\ell}\left(\varepsilon_{\ell}, \lambda_{\ell}, \lambda_{\ell+1}\right)=\left\|\psi_{\ell, n_{\ell+1}}^{\varepsilon_{\ell}}-\lambda_{\ell+1}\right\|_{L^{2}(\Omega)}^{2}+\delta t \sum_{j=n_{\ell}}^{n_{\ell+1}-1} \alpha_{\ell, j}^{\prime} \varepsilon_{\ell, j}^{2},
$$

with:

$$
\alpha_{\ell, j}^{\prime}=\frac{\alpha_{j}}{\beta_{\ell}}
$$


Note that the optimization problems defined on $\left[T_{\ell}, T_{\ell+1}\right]$ via $J_{\ell}$ are similar to the initial control problem on $[0, T]$ corresponding to (1.5).

3. Monotonic parareal algorithm. Our aim is to optimize $J_{\|}(\varepsilon, \Lambda)$ with respect to its two variables. We first present the main features of our algorithm and then give further details on each step.

3.1. Structure of the algorithm. To couple parareal framework and the control optimization, we propose the following methodology: given $\nu>0$, consider the termination criterion $c(\varepsilon)=J(\varepsilon)+\nu \sum_{\ell=0}^{N-2}\left|\varepsilon_{\ell, n_{\ell+1}-1}-\varepsilon_{\ell+1, n_{\ell+1}}\right|^{2}$. Given an initial control field $\varepsilon^{k}$ and a tolerance tol $\geq 0$, the computation of $\varepsilon^{k+1}$ is done as follows:

1. If $c\left(\varepsilon^{k}\right) \leq$ tol then stop.

2. Compute a coarse solution $\psi^{k}=\psi^{\varepsilon^{k}}$ of (1.6).

3. Compute a coarse solution $\chi^{k}=\chi^{\varepsilon^{k}}$ of (1.7).

4. Using $\psi^{k}$ and $\chi^{k}$, compute $\Lambda^{k}$ that optimizes $J_{\|}\left(\varepsilon^{k}, \Lambda\right)$ with respect to $\Lambda$.

5. On each interval $\left[T_{\ell}, T_{\ell+1}\right]$, compute in parallel some control field $\varepsilon_{\ell}^{k+1}$ that optimizes the cost functionals $J_{\ell}\left(\varepsilon_{\ell}, \lambda_{\ell}^{k}, \lambda_{\ell+1}^{k}\right)$ with respect to $\varepsilon_{\ell}$.

6. Define $\varepsilon^{k+1}$ as the concatenation of the control fields $\varepsilon_{\ell}^{k+1}$.

7. Assign $k \leftarrow k+1$. Return to Step 1 .

This algorithm is similar to an alternate direction descent algorithm, in the sense that it alternatively optimizes $J_{\|}(\varepsilon, \Lambda)$ with respect to $\Lambda$ and to $\varepsilon$.

Of course, to take advantage of the time parallelization, Steps 2 and 3 of the previous algorithm are to be computed using the coarse propagator,whereas the step 5 can be done simultaneously and by fine propagations.

REMARK 2. Solving (1.7) expoits in an essential manner the time-reversibility of the Schrödinger equation. Further work is required to extend this algorithm to non-reversible cases.

3.2. Virtual controls definition. We present in this section some results which will enable us to achieve efficiently the step 4 of the Monotonic parareal algorithm. As we do not intend to deal with the coarse propagator properties, we will consider in this section that Steps 2 and 3 are done with the split-operator method presented in section 2.1.2, with the (small) time step $\delta t$. Even though this is not what we want to do ultimately, the results below keep a practical interest since the most expensive part of the algorithm is the update of the control field which will be done in parallel. Thus, given a control field $\varepsilon=\left(\varepsilon_{j}\right)_{j=0, \ldots, n-1}$, the states $\psi^{\varepsilon}=\left(\psi_{j}^{\varepsilon}\right)_{j=0, \ldots, n}$ and the adjoint states $\chi^{\varepsilon}=\left(\chi_{j}^{\varepsilon}\right)_{j=0, \ldots, n}$ are computed by:

$$
\begin{gathered}
\left\{\begin{aligned}
\psi_{j+1}^{\varepsilon} & =e^{\frac{H_{0} \delta t}{2 i}} e^{\frac{V-\mu \varepsilon_{j}}{i} \delta t} e^{\frac{H_{0} \delta t}{2 i}} \psi_{j}^{\varepsilon} \\
\psi_{0}^{\varepsilon} & =\psi_{\text {init }},
\end{aligned}\right. \\
\left\{\begin{array}{c}
\chi_{j-1}^{\varepsilon}=e^{-\frac{H_{0} \delta t}{2 i}} e^{-\frac{V-\mu \varepsilon_{j}-1}{i} \delta t} e^{-\frac{H_{0} \delta t}{2 i}} \chi_{j}^{\varepsilon} \\
\chi_{n}^{\varepsilon}=\psi_{\text {target }} .
\end{array}\right.
\end{gathered}
$$

For reason of simplicity, we use in the sequel the following notation:

$$
\mathcal{F}_{j, j^{\prime}}^{\varepsilon} \psi_{j}^{\varepsilon}=\psi_{j^{\prime}}^{\varepsilon},
$$


where $0 \leq j, j^{\prime} \leq n$ are two (time) indices, and $\psi^{\varepsilon}=\left(\psi_{j}^{\varepsilon}\right)_{j=0, \ldots, n}$ is the solution of (3.1). We still denote by $J$ the time discretized cost functional corresponding to (1.5):

$$
J(\varepsilon)=\left\|\psi_{n}^{\varepsilon}-\psi_{\text {target }}\right\|_{L^{2}(\Omega)}^{2}+\delta t \sum_{j=0}^{n-1} \alpha_{j} \varepsilon_{j}^{2} .
$$

The following theorem provides the optimal choice of virtual controls $\Lambda$.

THEOREM 3.1. With the previous notations, let us define $\Lambda^{\varepsilon}=\left(\lambda_{\ell}^{\varepsilon}\right)_{\ell=1, \ldots, N-1}$ by:

$$
\lambda_{\ell}^{\varepsilon}=\left(1-\gamma_{\ell}\right) \psi_{n_{\ell}}^{\varepsilon}+\gamma_{\ell} \chi_{n_{\ell}}^{\varepsilon},
$$

where $\gamma_{\ell}=\frac{n_{\ell}}{n}$. Then :

$$
\Lambda^{\varepsilon}=\operatorname{argmin}_{\Lambda}\left(J_{\|}(\varepsilon, \Lambda)\right) .
$$

Moreover we have:

$$
J_{\|}\left(\varepsilon, \Lambda^{\varepsilon}\right)=J(\varepsilon)
$$

Proof. : For a given $\Lambda=\left(\lambda_{\ell}\right)_{\ell=1, \ldots, N-1}$, let us first prove that $J(\varepsilon)$ is a lower bound for $J_{\|}(\varepsilon, \Lambda)$. The Cauchy-Schwartz inequality gives :

$$
\begin{aligned}
\sum_{\ell=0}^{N-1} \beta_{\ell}\left\|\psi_{\ell, n_{\ell+1}}^{\varepsilon_{\ell}}-\lambda_{\ell+1}\right\|_{L^{2}(\Omega)}^{2} & =\left(\sum_{\ell=0}^{N-1} \frac{1}{\beta_{\ell}}\right) \sum_{\ell=0}^{N-1} \beta_{\ell}\left\|\psi_{\ell, n_{\ell+1}}^{\varepsilon_{\ell}}-\lambda_{\ell+1}\right\|_{L^{2}(\Omega)}^{2} \\
& \geq\left(\sum_{\ell=0}^{N-1}\left\|\psi_{\ell, n_{\ell+1}}^{\varepsilon_{\ell}}-\lambda_{\ell+1}\right\|_{L^{2}(\Omega)}\right)^{2} .
\end{aligned}
$$

Recalling that $\mathcal{F}^{\varepsilon}$ is a unitary operator, we have:

$\forall \ell=1, \ldots, N-1$

$$
\begin{aligned}
\left\|\psi_{\ell, n_{\ell+1}}^{\varepsilon}-\lambda_{\ell+1}\right\|_{L^{2}(\Omega)} & =\left\|\mathcal{F}_{n_{\ell, n_{\ell+1}}^{\varepsilon}}^{\varepsilon}\left(\lambda_{\ell}\right)-\lambda_{\ell+1}\right\|_{L^{2}(\Omega)} \\
& =\left\|\mathcal{F}_{n_{\ell+1}, n}^{\varepsilon}\left(\mathcal{F}_{n_{\ell}, n_{\ell+1}}^{\varepsilon}\left(\lambda_{\ell}\right)-\lambda_{\ell+1}\right)\right\|_{L^{2}(\Omega)} \\
& =\left\|\mathcal{F}_{n_{\ell}, n}^{\varepsilon}\left(\lambda_{\ell}\right)-\mathcal{F}_{n_{\ell+1}, n}^{\varepsilon}\left(\lambda_{\ell+1}\right)\right\|_{L^{2}(\Omega)} .
\end{aligned}
$$

Hence:

$$
\begin{aligned}
\sum_{\ell=0}^{N-1}\left\|\psi_{\ell, n_{\ell+1}}^{\varepsilon \ell}-\lambda_{\ell+1}\right\|_{L^{2}(\Omega)} & =\sum_{\ell=0}^{N-1}\left\|\mathcal{F}_{n_{\ell}, n}^{\varepsilon}\left(\lambda_{\ell}\right)-\mathcal{F}_{n_{\ell+1}, n}^{\varepsilon}\left(\lambda_{\ell+1}\right)\right\|_{L^{2}(\Omega)} \\
& \geq\left\|\mathcal{F}_{0, n}^{\varepsilon}\left(\psi_{\text {init }}\right)-\psi_{\text {target }}\right\|_{L^{2}(\Omega)} .
\end{aligned}
$$

Combining (3.11) and (3.8) we obtain, since $\mathcal{F}_{0, n}^{\varepsilon}\left(\psi_{\text {init }}\right)=\psi_{n}^{\varepsilon}$ :

$$
J_{\|}(\varepsilon, \Lambda) \geq J(\varepsilon) .
$$


By (3.5), we have:

$$
\psi_{\ell, n_{\ell+1}}^{\varepsilon_{\ell}}-\lambda_{\ell+1}^{\varepsilon}=\mathcal{F}_{n_{\ell}, n_{\ell+1}}^{\varepsilon}\left(\lambda_{\ell}^{\varepsilon}\right)-\lambda_{\ell+1}^{\varepsilon}
$$

$$
\begin{aligned}
& =\mathcal{F}_{n_{\ell}, n_{\ell+1}}^{\varepsilon}\left(\left(1-\gamma_{\ell}\right) \psi_{n_{\ell}}^{\varepsilon}+\gamma_{\ell} \chi_{n_{\ell}}^{\varepsilon}\right)-\left(\left(1-\gamma_{l+1}\right) \psi_{n_{\ell+1}}^{\varepsilon}+\gamma_{\ell+1} \chi_{n_{\ell+1}}^{\varepsilon}\right) \\
& =\left(\gamma_{\ell+1}-\gamma_{\ell}\right)\left(\psi_{n_{\ell+1}}^{\varepsilon}-\chi_{n_{\ell+1}}^{\varepsilon}\right) .
\end{aligned}
$$

Hence,

$$
\left\|\psi_{\ell, n_{\ell+1}}^{\varepsilon_{\ell}}-\lambda_{\ell+1}^{\varepsilon}\right\|_{L^{2}(\Omega)}=\frac{1}{\beta_{\ell}}\left\|\psi_{n}^{\varepsilon}-\psi_{\text {target }}\right\|_{L^{2}(\Omega)}
$$

Combining this equality with (2.5) we obtain:

$$
\begin{aligned}
J_{\|}\left(\varepsilon, \Lambda^{\varepsilon}\right) & =\sum_{\ell=0}^{N-1} \frac{1}{\beta_{\ell}}\left\|\psi_{n}^{\varepsilon}-\psi_{\text {target }}\right\|_{L^{2}(\Omega)}^{2}+\delta t \sum_{\ell=0}^{N-1} \sum_{j=n_{\ell}}^{n_{\ell+1}-1} \alpha_{j} \varepsilon_{\ell, j}^{2}, \\
& =\left\|\psi_{n}^{\varepsilon}-\psi_{\text {target }}\right\|_{L^{2}(\Omega)}^{2}+\delta t \sum_{\ell=0}^{N-1} \sum_{j=n_{\ell}}^{n_{\ell+1}-1} \alpha_{j} \varepsilon_{\ell, j}^{2},
\end{aligned}
$$

and the theorem follows.

REMARK 3. The trajectory $\left(\left(1-\frac{j}{N}\right) \psi_{j}^{\varepsilon}+\frac{j}{N} \chi_{j}^{\varepsilon}\right)_{j=0, \ldots, n}$ is an ideal trajectory that reaches exactly the target $\psi_{\text {target }}$. The choice $\Lambda=\Lambda^{\varepsilon}$ is equivalent to define the virtual controls on this trajectory. This interpretation is closely related to the concept of Reference trajectory tracking: through the introduction of the parareal cost functionals, the initial problem is transformed into $N-1$ optimization problems that aim to minimize on each subinterval the distance between the current trajectory and this ideal (unknown) trajectory.

REMARK 4. An alternative definition for $\Lambda$ can be:

$$
\lambda_{\ell}=\frac{\left(1-\gamma_{\ell}\right) \psi_{n_{\ell}}^{\varepsilon}+\gamma_{\ell} \chi_{n_{\ell}}^{\varepsilon}}{\left\|\left(1-\gamma_{\ell}\right) \psi_{n_{\ell}}^{\varepsilon}+\gamma_{\ell} \chi_{n_{\ell}}^{\varepsilon}\right\|_{L^{2}(\Omega)}},
$$

that has the advantage that the norms of the virtual controls are preserved. Furthermore, it can be proved that $\lambda$ corresponds to a critical point of $J_{\|}(\varepsilon, \Lambda)$ under the constraint:

$$
\forall \ell=1, \ldots, N-1,\left\|\lambda_{\ell}\right\|_{L^{2}(\Omega)}=1 .
$$

3.3. Monotonic schemes. Let us now describe a practical implementation of Step 5 of the Monotonic parareal algorithm. Given a set of virtual controls $\Lambda=$ $\left(\lambda_{\ell}\right)_{\ell=1, \ldots, N-1}$ (recall that $\lambda_{0}=\psi_{0}$ and $\left.\lambda_{N}=\psi_{\text {target }}\right)$, the parareal cost functional $J_{\ell}$ reads:

$$
\begin{aligned}
J_{\ell}\left(\varepsilon_{\ell}, \lambda_{\ell}, \lambda_{\ell+1}\right)= & \left\|\lambda_{\ell}\right\|_{L^{2}(\Omega)}^{2}+\left\|\lambda_{\ell+1}\right\|_{L^{2}(\Omega)}^{2}-2 R e\left\langle\psi_{\ell, n_{\ell+1}}, \lambda_{\ell+1}\right\rangle \\
& +\delta t \sum_{j=n_{\ell}}^{n_{\ell+1}-1} \alpha_{\ell, j}^{\prime} \varepsilon_{\ell, j}^{2}, \quad \ell=0, \ldots, N-1 .
\end{aligned}
$$


The first two terms of $J_{\ell}$ will not change during the optimization of $\varepsilon$. An efficient way to minimize cost functionals of the form $\widetilde{J}(\varepsilon)=-2 R e\left\langle\psi(T), \psi_{\text {target }}\right\rangle+\int_{0}^{T} \alpha(t) \varepsilon^{2}(t) d t$ associated with the Schrödinger equation is to use monotonic schemes ([21],[37]).

In our case, the time discretized monotonic scheme corresponding to (3.18) can be defined by the following procedure.

Let us consider $(\delta, \eta) \in[0,2[\times[0,2[$ and introduce the notations:

$$
\tilde{\chi}_{\ell, j}=e^{\frac{H_{0} \delta t}{2 i}} \chi_{\ell, j}, \breve{\psi}_{\ell, j}=e^{\frac{H_{0} \delta t}{2 i}} \psi_{\ell, j}, \breve{\chi}_{\ell, j}=e^{-\frac{H_{0} \delta t}{2 i}} \chi_{\ell, j}, \widetilde{\psi}_{\ell, j}=e^{-\frac{H_{0} \delta t}{2 i}} \psi_{\ell, j},
$$

and $\mu^{*}(h)$, an approximation of $\mu$, defined by:

$$
\mu^{*}(h)=\frac{e^{-i \mu h \delta t}-I d}{-i h \delta t},
$$

where $I d$ is the identity operator.

Given a control field $\varepsilon^{k}$, its restriction $\varepsilon_{\ell}^{k}$ to the interval $\left[T_{\ell}, T_{\ell+1}\right]$ and the corresponding $\psi_{\ell}^{k}=\left(\psi_{\ell, j}^{k}\right)_{j=n_{\ell}, \ldots, n_{\ell+1}}$ are defined iteratively by:

$$
\left\{\begin{aligned}
\psi_{\ell, j+1}^{k} & =e^{\frac{H_{0} \delta t}{2 i}} e^{\frac{V-\mu \varepsilon_{\ell, j}^{k}}{i} \delta t} e^{\frac{H_{0} \delta t}{2 i}} \psi_{\ell, j}^{k}, \quad j=n_{\ell}, \ldots, n_{\ell+1}-1, \\
\psi_{\ell, n_{\ell}}^{k} & =\lambda_{\ell}, \quad \ell=0, \ldots, N-1 .
\end{aligned}\right.
$$

The computation of $\varepsilon_{\ell}^{k+1}$ is performed as follows:

1. For $\ell=0, \ldots, N-1$, compute iteratively an intermediate control field $\widetilde{\varepsilon}_{\ell}^{k}$ and its corresponding adjoint state $\chi_{\ell}^{k}$ by:

$$
\chi_{\ell, j}^{k}=e^{-\frac{H_{0} \delta t}{2 i}} e^{\frac{-V+\mu \tilde{\varepsilon}_{\ell, j}^{k}}{i} \delta t} e^{-\frac{H_{0} \delta t}{2 i}} \chi_{\ell, j+1}^{k}, \quad j=n_{\ell}, \ldots, n_{\ell+1}-1,
$$

where $\widetilde{\varepsilon}_{\ell, j}^{k}$ is such that:

$$
\widetilde{\varepsilon}_{\ell, j}^{k}=(1-\eta) \varepsilon_{\ell, j}^{k}-\frac{\eta}{\alpha_{\ell, j}^{\prime}} \operatorname{Im}\left\langle\breve{\chi}_{\ell, j+1}^{k}\left|\mu^{*}\left(\varepsilon_{\ell, j}^{k}-\widetilde{\varepsilon}_{\ell, j}^{k}\right)\right| \widetilde{\psi}_{\ell, j+1}^{k}\right\rangle
$$

with the final condition:

$$
\chi_{\ell, n_{\ell+1}}^{k}=\lambda_{\ell+1} \text {. }
$$

2. For $\ell=0, \ldots, N-1$, compute iteratively the control field $\varepsilon_{\ell}^{k+1}$ and its corresponding state $\psi_{\ell}^{k+1}$ by:

$$
\psi_{\ell, j+1}^{k+1}=e^{\frac{H_{0} \delta t}{2 i}} e^{\frac{V-\mu \varepsilon_{\ell, j}^{k+1}}{i} \delta t} e^{\frac{H_{0} \delta t}{2 i}} \psi_{\ell, j}^{k+1}, \quad j=n_{\ell}, \ldots, n_{\ell+1}-1,
$$

where $\varepsilon_{\ell, j}^{k+1}$ is such that:

$$
\varepsilon_{\ell, j}^{k+1}=(1-\delta) \widetilde{\varepsilon}_{\ell, j}^{k}-\frac{\delta}{\alpha_{\ell, j}^{\prime}} \operatorname{Im}\left\langle\widetilde{\chi}_{j}^{k}\left|\mu^{*}\left(\varepsilon_{\ell, j}^{k+1}-\widetilde{\varepsilon}_{\ell, j}^{k}\right)\right| \breve{\psi}_{\ell, j}^{k+1}\right\rangle,
$$

with the initial condition:

$$
\psi_{\ell, n_{\ell}}^{k+1}=\lambda_{\ell} .
$$


The implicit equations (3.23) and (3.26) are solved independently for $\widetilde{\varepsilon}_{\ell, j}^{k}$ and $\varepsilon_{\ell, j}^{k+1}$ respectively at each time step by a Newton method (all others parameters involved are known). We refer the reader to [20] for a proof of existence of solutions and further details on this scheme.

In the sequel, the initial value $\varepsilon^{0}$ of the monotonic scheme is considered fixed. An important property of this algorithm is given in the following theorem [21]:

TheOREm 3.2. For any $\eta, \delta \in[0,2]$ the algorithm given in Eqns. (3.22)-(3.27) is well defined and converges monotonically in the sense that:

$$
\begin{aligned}
& \forall \ell=0, \ldots, N-1 \\
& J_{\ell}\left(\varepsilon_{\ell}^{k+1}, \lambda_{\ell}, \lambda_{\ell+1}\right)-J_{\ell}\left(\varepsilon_{\ell}^{k}, \lambda_{\ell}, \lambda_{\ell+1}\right)=-\delta t \sum_{j=n_{\ell}}^{n_{\ell+1}-1} \alpha_{\ell, j}^{\prime}\left(\varepsilon_{j, \ell}^{k+1}-\varepsilon_{j, \ell}^{k}\right)^{2} \leq 0 .
\end{aligned}
$$

This optimization procedure can be done in parallel on each interval $\left[T_{\ell}, T_{\ell+1}\right]$. Consequently, the computations can be carried out with fine propagators $\mathcal{F}_{\ell}^{\varepsilon_{\ell}^{k+1}}$.

REMARK 5. As it was the case for Step 4 (see Remark 3), this step of the algorithm is also linked to the concept of Reference trajectory tracking: in the monotonic schemes, the control field $\widetilde{\varepsilon}_{\ell, j 1}^{k}$ (resp. $\varepsilon_{\ell, j}^{k+1}$ ) is chosen such that the distance between the current states and adjoint state $\left\|\psi_{\ell, j}^{k}-\chi_{\ell, j}^{k}\right\|_{L^{2}(\Omega)} \quad\left(\right.$ resp $\left\|\psi_{\ell, j+1}^{k+1}-\chi_{\ell, j+1}^{k}\right\|_{L^{2}(\Omega)}$ ) decreases at each time step. We refer the reader to [28, 32] for details about the relationship between the monotonic schemes and local tracking algorithms.

REMARK 6. Note that several iterations of this scheme can be done during the step 5 of the Monotonic parareal algorithm, especially in case of slow convergence (see Table 5.1 in Section 5.4 for numerical results about it).

The algorithm is schematically depicted in Fig. 3.1.

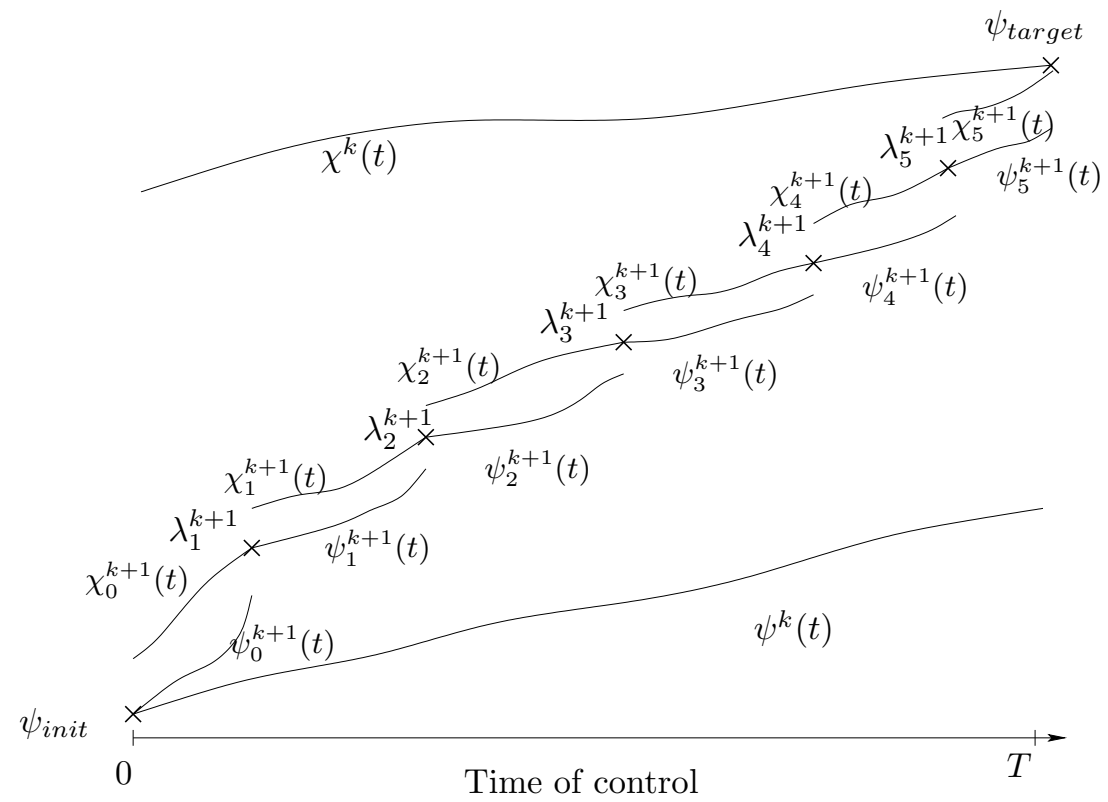

FIG. 3.1. Symbolic representation of one iteration of the Monotonic parareal algorithm. The optimization is achieved in parallel on each subinterval $\left[T_{\ell}, T_{\ell+1}\right]$. The virtual controls $\lambda_{\ell}^{k}$ are updated at each iteration. 
3.4. Monotonicity of the algorithm. The combination of the two above strategies allows to define:

$$
\Lambda^{k}=\Lambda^{\varepsilon^{k}}
$$

and $\varepsilon^{k+1}$ as the concatenation of the sequence $\left(\varepsilon_{\ell}^{k+1}\right)_{\ell=0, \ldots, N-1}$. We have thus obtained a global monotonic algorithm since:

$$
J\left(\varepsilon^{k+1}\right)=J_{\|}\left(\varepsilon^{k+1}, \Lambda^{k+1}\right) \leq J_{\|}\left(\varepsilon^{k+1}, \Lambda^{k}\right) \leq J_{\|}\left(\varepsilon^{k}, \Lambda^{k}\right)=J\left(\varepsilon^{k}\right) .
$$

4. Convergence of the algorithm. The convergence of the sequence $\left(\varepsilon^{k}\right)_{k \in \mathbf{N}}$ defined by the above algorithm is described in the next theorem.

THEOREM 4.1. Given an initial control field $\varepsilon^{0}$, consider the sequence $\left(\varepsilon^{k}\right)_{k \in \boldsymbol{N}}$ obtained by the algorithm (3.22)-(3.27), where the coarse propagator in Steps 2-3 is taken to be the same as the fine propagator. The sequence $\left(\varepsilon^{k}\right)_{k \in \boldsymbol{N}}$ converges towards a critical point of $J$.

Proof. : Since the proof is very similar to that presented in [26], we only give a brief overview.

Denote by $C_{J}$ the set of critical points of $J$. Using the previous notations, this set reads:

$$
C_{J}=\left\{\varepsilon / \forall j=0, \ldots, N-1, \operatorname{Im}\left\langle\widetilde{\chi}_{j}^{\varepsilon}|\mu| \breve{\psi}_{j}^{\varepsilon}\right\rangle+\alpha_{j} \varepsilon_{j}=0\right\} .
$$

Let $C_{\varepsilon^{0}}$ be the set of limit points of $\left(\varepsilon^{k}\right)_{k \in \mathbf{N}}$.

Since $\left\|\lambda_{0}^{k}\right\|_{L^{2}(\Omega)}=\left\|\lambda_{N}^{k}\right\|_{L^{2}(\Omega)}=1$, equation (3.5) implies that :

$$
\forall \ell=0, \ldots, N, \quad\left\|\lambda_{\ell}^{k}\right\|_{L^{2}(\Omega)} \leq 1 .
$$

It can then be proved by induction that (see [20], Theorem 3):

$$
\forall k \in \mathbf{N}, \forall j=0, \ldots, n-1,\left|\varepsilon_{j}^{k}\right| \leq M,
$$

with:

$$
M=\max \left(\left\|\varepsilon^{0}\right\|_{\infty}, \max \left(1, \frac{\delta}{2-\delta}, \frac{\eta}{2-\eta}\right) \frac{\|\mu\|_{*}}{\min _{j=1, . ., n-1}\left(\alpha_{j}\right)}\right)
$$

where $\|\mu\|_{*}$ denotes the operator norm of $\mu$ and $\left\|\varepsilon^{0}\right\|_{\infty}=\max _{j=0, \ldots, n-1}\left(\left|\varepsilon_{j}^{0}\right|\right)$. Consider now a converging subsequence $\left(\varepsilon^{k_{p}}\right)_{p \in \mathbf{N}}$ and its limit $\varepsilon^{\infty} \in C_{\varepsilon^{0}}$. The corresponding state $\psi^{\varepsilon_{k_{p}}}=\psi^{k_{p}}$ and adjoint state $\chi^{\varepsilon_{k_{p}}}=\chi^{k_{p}}$ defined by (3.1) and (3.2) converge respectively towards $\psi^{\varepsilon^{\infty}}$ and $\chi^{\varepsilon^{\infty}}$. By $(3.5)$, the sequence $\left(\Lambda_{p}^{k}\right)_{p \in \mathbf{N}}$ converges towards $\Lambda^{\varepsilon^{\infty}}$. Then, a similar proof indicates that $\left(\psi_{\ell}^{k_{p}}\right)_{p \in \mathbf{N}}$ and $\left(\chi_{\ell}^{k_{p}}\right)_{p \in \mathbf{N}}$ converge towards $\psi_{\ell}^{\varepsilon^{\infty}}$ and $\chi_{\ell}^{\varepsilon^{\infty}}$. Thanks to the similar structures of $J_{\ell}$ and $J$, one can adapt the proof of Lemma 3.3 in [26] that shows that:

$$
\forall \ell=0, \ldots, N-1, \forall j=n_{\ell}, \ldots, n_{\ell+1}-1, \operatorname{Im}\left\langle\widetilde{\chi}_{\ell, j}^{\varepsilon^{\infty}}|\mu| \breve{\psi}_{\ell, j}^{\varepsilon^{\infty}}\right\rangle+\alpha_{\ell, j}^{\prime} \varepsilon_{\ell, j}^{\infty}=0
$$

Another use of (3.5) proves that:

$(4.6) \forall \ell=0, \ldots, N-1, \forall j=n_{\ell}, \ldots, n_{\ell+1}-1, \frac{1}{\beta_{\ell}} \operatorname{Im}\left\langle\widetilde{\chi}_{\ell, j}^{\varepsilon^{\infty}}|\mu| \breve{\psi}_{\ell, j}^{\varepsilon^{\infty}}\right\rangle=\operatorname{Im}\left\langle\widetilde{\chi}_{j}^{\varepsilon^{\infty}}|\mu| \breve{\psi}_{j}^{\varepsilon^{\infty}}\right\rangle$. 
Combining (4.5) and (4.6) with (2.8), we obtain that:

$$
C_{\varepsilon^{0}} \subset C_{J} .
$$

Since $\left(J\left(\varepsilon^{k}\right)\right)_{k \in \mathbf{N}}$ is a bounded (by $2+\max _{j=0, \ldots, n-1}\left(\alpha_{j}\right) T M$ ) monotonic sequence, it converges towards a limit denoted by $l_{\varepsilon^{0}}$.

We are now in the position to reproduce the analysis in Theorem 4.5 of [26] that shows that:

$$
\begin{aligned}
& \left.\exists \theta \in] 0, \frac{1}{2}\right], \quad \exists \kappa>0, \quad \exists k_{0} \in \mathbf{N} / \forall k \geq k_{0}, \\
& \left(l_{\varepsilon^{0}}-J\left(\varepsilon^{k}\right)\right)^{\theta}-\left(l_{\varepsilon^{0}}-J\left(\varepsilon^{k+1}\right)\right)^{\theta} \geq \kappa\left\|\varepsilon^{k+1}-\varepsilon^{k}\right\|_{2},
\end{aligned}
$$

where $\|\cdot\|_{2}$ denotes the usual euclidean norm on $\mathbf{R}^{n}$. Hence, the sequence $\left(\varepsilon^{k}\right)_{k \in \mathbf{N}}$ is a Cauchy sequence and by (4.7) the theorem follows. $\square$

\section{Numerical results.}

5.1. Model. In order to test the performance of the algorithm, a case already treated in the literature has been considered. The system is a molecule of $H C N$ modelled as a rigid rotator. We refer the reader to $[1,27]$ for numerical details concerning this system. The goal is to control the orientation of the system ; this is expressed through the requirement that $\left\|\psi(T)-\psi_{\text {target }}\right\|_{L^{2}(\Omega)}$ is minimized, where the target function $\psi_{\text {target }}$ corresponds to an orientated state.

5.2. Propagators. All the propagations are done through a Strang-secondorder split-operator type, as e.g. in (3.21). The coarse propagator, corresponding to (3.1) and (3.2), is used with a large time step, whereas the fine propagator, as it appears in (3.26) and (3.23), is used with a small time step. The ratio of these two time steps is 10 . We have observed that a renormalization (3.16) slightly reduces the speed of convergence, but has no effect on the converged control fields.

5.3. Numerical convergence. Let us present some results conerning the convergence of the Monotonic parareal algorithm.

5.3.1. Evolution of $\left(\varepsilon^{k}\right)_{k \in \mathbf{N}}$. In a first test, $N=10$ identical subintervals are considered to parallelize the algorithm. Figures 5.1-5.3 show a typical evolution of the sequence $\left(\varepsilon^{k}\right)_{k \in \mathbf{N}}$ computed by our algorithm. Figure 5.4 represents the control field obtained without parallelization by a monotonic algorithm applied to $J$.

Note that the discontinuities that are visible on Figure 5.1 and even on Figure 5.2 disappear as the number of iteration increases.

5.3.2. Variation of the number of subintervals. The control field obtained at the numerical convergence appears to be independent of the number $N$ of subintervals. This is coherent with Theorem 4.1 which claims that the limit only depends on the initial cost functional $J$. In order to evaluate the effect of $N$ on the convergence of the algorithm, we have run the algorithm for different values of this parameter. Figure 5.5 shows the evolution of the cost functional values for $N=1,2,5,10,50,100$. The convergence speed decreases when $N$ becomes larger. One has thus to find a optimum between the acceleration obtained by parallelization and the reduction of the convergence speed induced by it. This compromise depends of the choice of the coarse and fine propagators. In our case, the parallel optimizations and the coarse propagations allow to reach a satisfactory cost functional value with an actual gain 


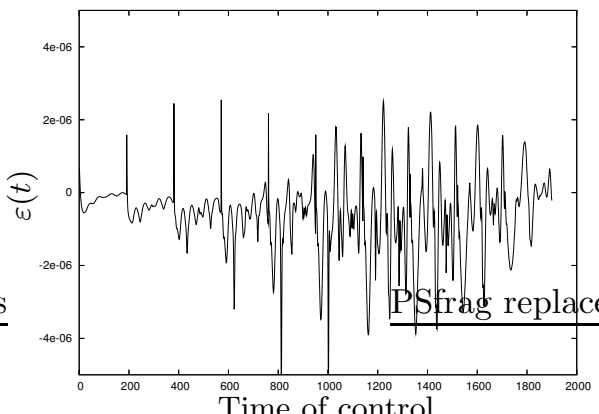

FIG. 5.1. Field of control obtained after one iteration.

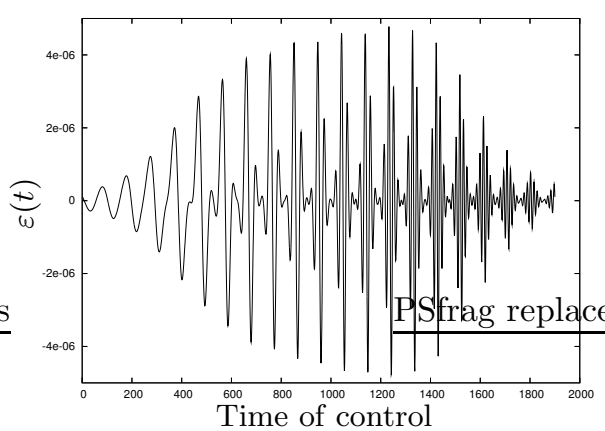

FIG. 5.3. Field of control obtained after 250 iterations. In such nonlinear the typical number of iterations is in the range $10^{2}--10^{4}$ $[1,27]$

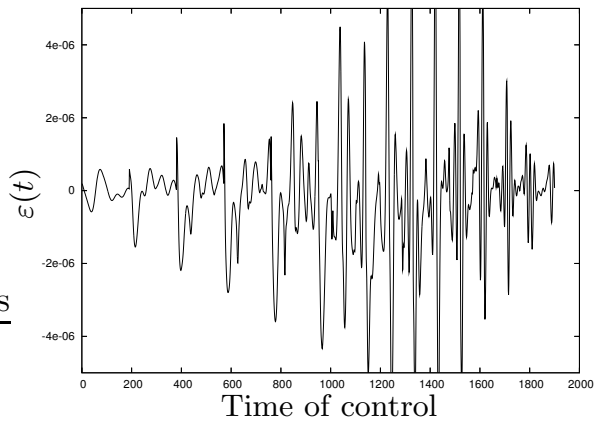

FIG. 5.2. Field of control obtained after 10 iterations.

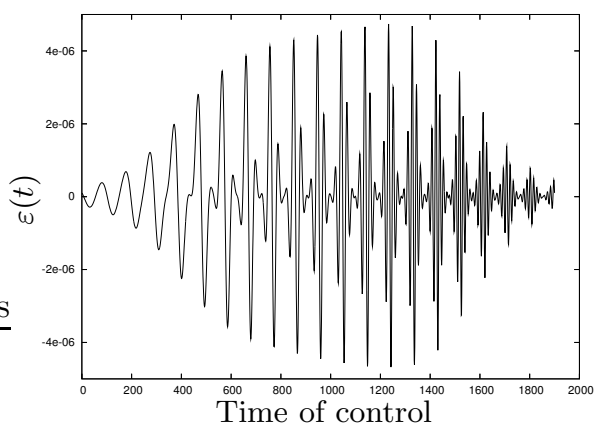

Fig. 5.4. Field of control obtained by a non-parallelized monotonic algorithm (i.e. with $N=1)$ after 250 iterations.

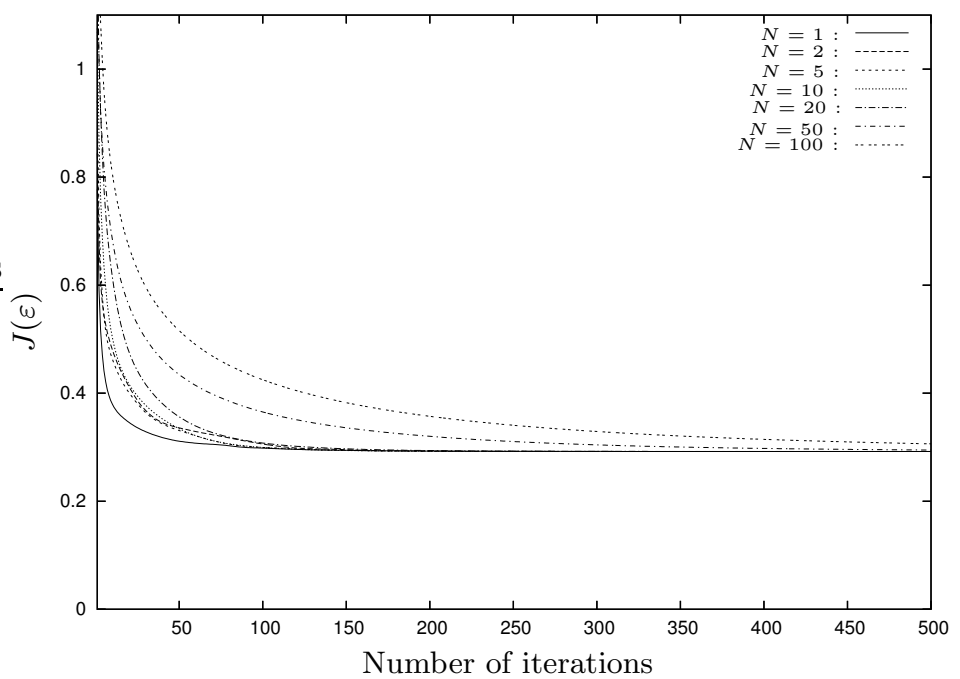

FIG. 5.5. Evolution of the cost functional value during the first 500 iterations.

in "wall-clock" time roughly equals to 7 . We have not sought to optimize the coarse 


\begin{tabular}{|c|c|c|c|c|}
\hline & $k$ & $m$ & Comp. Time & $J\left(\varepsilon^{k}\right)$ \\
\hline case 0 & 100 & 1 & $100.10 . T_{f}$ & 0.2983 \\
\hline case 1 & 100 & 1 & $100 .\left(T_{f}+T_{C}\right)$ & 0.2986 \\
\hline case 2 & 50 & 2 & $50 .\left(2 T_{f}+T_{C}\right)$ & 0.3062 \\
\hline case 3 & 25 & 4 & $25 .\left(4 T_{f}+T_{C}\right)$ & 0.3295 \\
\hline
\end{tabular}

Results obtained for several subiterations of the monotonic schemes (3.22)-(3.27) during Step 5 of the Monotonic parareal algorithm.

and the fine propagators for these numerical tests. In particular, a coarser propagator should improve this result.

5.4. About the full efficiency. Consider again $N=10$ identical subintervals. An ideal time parallelization should divide the computational time by 10 . In order to approach such a full efficiency, a strategy would be to increase the parallel computations (step 5) with respect to the coarse propagations (steps 2 and 3 of the algorithm). In this perspective, the influence on the convergence of the number of iterations (denoted by $m$ ) of the monotonic algorithm (3.23)-(3.26) done during the step 5 has been tested (see Remark 6 ). Let denote by $k$ the number of iterations of the Monotonic parareal algorithm, $T_{f}$ the computational time of one iteration of the monotonic algorithm in a subinterval and $T_{C}$ the computational time of both step 2 and step 3. Table 5.1 summarize the cases that have been tested.

Case 0 corresponds to the non-parallelized monotonic algorithm (i.e. with $\mathrm{N}=1$ ) computed with the fine propagator. Figure 5.6 shows that the best strategy corresponds to the case 1. Further work is needed to reach the full efficiency (corresponding in our case to a computational time close to $\left.100 . T_{f}\right)$.

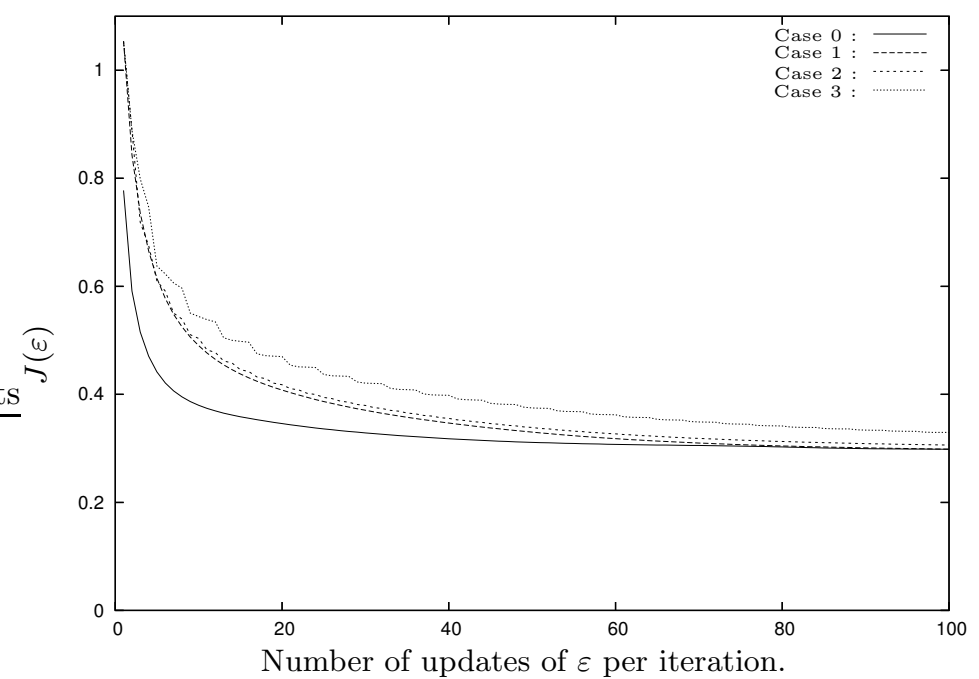

FIG. 5.6. Evolutions of the cost functional values for different values of $m$. 


\section{REFERENCES}

[1] A. Auger, A. Ben Haj Yedder, E. Cances, C. Le Bris, C. M. Dion, A. Keller, and O. AтAвек, Optimal laser control of molecular systems: methodology and results, Mathematical Models and Methods in Applied Sciences, 12 (2002), pp. 1281-1315.

[2] L. Baffico, S. Benard, Y. Maday, G. Turinici, and G. Zérah, Parallel in time molecular dynamics simulations, Phys. Rev. E., 66 :057701 (2002).

[3] A. D. Bandrauk And H. Shen, Exponential split operator methods for solving coupled timedependent Schrödinger equations, J. Chem. Phys., 99 (1993), pp. 1185-1193.

[4] A Bonzí, Multigrid methods for parabolic distributed optimal control problems, J. Comp. App. Math., 157 (2003), pp. 365-382.

[5] T. Brixner, N. H. Damrauer, P. Niklaus, and G. Gerber, Photoselective adaptive femtosecond quantum control in the liquid phase, Nature, 414 (2001), pp. 57-60.

[6] T. Brixner, G. Krampert, T. Pfeifer, R. Selle, G. Gerber, M. Wollenhaupt, O. Graefe, C. Horn, D. Liese, and T. Baumert, Quantum control by ultrafast polarization shaping, Phys. Rev. Lett., 92: 208301 (2004).

[7] E. Brown and H. Rabitz, Some mathematical and algorithmic challenges in the control of quantum dynamics phenomena, J. Math. Chem., 31 (2002), pp. 17-63.

[8] P. Chartier and B. Philippe, A parallel shooting technique for solving dissipative ODEs, Computing, 51 (1993), pp. 209-236.

[9] F. Courvoisier, V. Boutou, J. Kasparian, E. Salmon, G. Méjean, J. Yu, and J-P. Wolf, Ultraintense light filaments transmitted through clouds, App. Phys. Lett., 83 (2003), pp. 213-215.

[10] M. Gander and S. Vandewalle, Analysis of the parareal time-parallel time-integration method, SI. Sci. Comp., in print, (2006).

[11] W. Hackbusch, Parabolic multi-grid methods, in Computing Methods in Applied Sciences and Engineering, VI (R. Glowinski and J.-L. Lions, eds.), 1984, pp. 189-197.

[12] - Multigrid methods and applications, Springer-Verlag, 1985.

[13] M. Heinkenschloss, A time-domain decomposition iterative method for the solution of distributed linear quadratic optimal control problems, J. Comp. App. Math., 173 (2005), pp. $169-198$.

[14] G. Horton, The time-parallel multigrid method, Comm. Appl. Numer. Methods, 8 (1992), pp. 585-595.

[15] R.S. Judson and H. Rabitz, Teaching lasers to control molecules, Phys. Rev. Lett, 68 (1992), pp. $1500-1503$.

[16] J. Kasparian, M. Rodriguez, G. MéJean, J. Yu, E. Salmon, H. Wille, R. Bourayou, S. Frey, Y.-B. Andr, A. Mysyrowicz, R. Sauerbrey, J.-P. Wolf, and L. Wöste, White-light filaments for atmospheric analysis, Science, 301 (2003), pp. 61-64.

[17] J. E. Lagnese And G. Leugering, Time-domain decomposition of optimal control problems for the wave equation, Systems Control Lett., 48 (2003), pp. 229-242.

[18] J-L. Lions, Y. MADAY, AND G. TuRINici, A parareal in time discretization of PDE, C. R. Acad. Sci, 332 (7) (2001), pp. 661-668.

[19] C. Lubich And A. Ostermann, Multi-grid dynamic iteration for parabolic equations, BIT, 27 (1987), pp. 216-234.

[20] Y. Maday, J. Salomon, and G. Turinici, Monotonic time-discretized schemes in quantum control, Num. Math., 103 (2006), pp. 323-338.

[21] Y. Maday and G. TURINICI, New formulations of monotonically convergent quantum control algorithms, J. Chem. Phys, 118 (2003).

[22] W.H. Press et Al, Numerical Recipes in C: The Art of Scientific Computing (2nd edn), Cambridge University Press, 2002.

[23] H. Rabitz R. J. Levis, G. M. Menkir, Selective bond dissociation and rearrangement with optimally tailored, strong-field laser pulses, Science, 292 (2001), pp. 709-713.

[24] H. Rabitz, R. de Vivie-Riedle, M. Motzkus, and K. Kompa, Whither the future of controlling quantum phenomena?, Science, 288 (2000), pp. 824-828.

[25] H. Rabitz, G. Turinici, And E. Brown, Control of quantum dynamics: Concepts, procedures and future prospects, In Ph. G. Ciarlet, editor, Computational Chemistry, Special Volume (C. Le Bris Editor) of Handbook of Numerical Analysis, vol X, Elsevier Science B.V., 2003.

[26] J. SAlomon, Convergence of the time-dicretized monotonic schemes, to appear in M2AN, (2006).

[27] J. Salomon, C. Dion, And G. Turinici, Optimal molecular alignment and orientation through rotational ladder climbing, J. Chem. Phys., 123: 144310 (2005).

[28] J. Salomon and G. Turinici, On the relationship between the local tracking procedures and 
monotonic schemes in quantum optimal control, J. Chem. Phys., 124: 074102 (2006).

[29] S. Shi, A. Woody, And H. Rabitz, Optimal control of selective vibrational excitation in harmonic linear chain molecules, J. Chem. Phys., 88 (1988), pp. 6870-6883.

[30] G. Strang, Accurate partial difference methods I: Linear cauchy problems, Arch. Rat. Mech. and An., 12 (1963), pp. 392-402.

[31] D. Tannor, V. Kazakov, and V. Orlov, Control of photochemical branching: Novel procedures for finding optimal pulses and global upper bounds, in Time Dependent Quantum Molecular Dynamics, Broeckhove J. and Lathouwers L., eds., Plenum, 1992, pp. 347-360.

[32] G. TuRINICI, Equivalence between local tracking procedures and monotonic algorithms in quantum control, in Proceedings of the 44th IEEE Conference on Decision and Control, Sevilla, Spain, December 2005, pp. 8203-8208.

[33] G. TURINICI AND Y MADAY, A parallel in time approach for quantum control : the parareal algorithm, in Proceedings of the 41th IEEE Conference on Decision and Control, Las Vegas, Nevada, USA, December 2002, pp. 62-66.

[34] S. UlbRICH, Generalized sqp-methods with "parareal" time-domain decomposition for timedependent pde-constrained optimization, accepted for publication, (2006).

[35] S. Vandewalle and R. Piessens, Efficient parallel algorithms for solving initial-boundary value and time-periodic parabolic partial differential equations, SIAM J. Sci. Stat. Comput., 13 (1992), pp. 1330-1346.

[36] G. Vogt, G. Krampert, P. Niklaus, P. Nuernberger, and G. Gerber, Optimal control of photoisomerization, Phys. Rev. Lett., 94: 68305 (2005).

[37] W. Zhu AND H. RABITZ, A rapid monotonically convergent iteration algorithm for quantum optimal control over the expectation value of a positive definite operator, J. Chem. Phys., 109 (1998), pp. 385-391. 